\title{
Evolving applications of microarray technology in postnatal diagnosis (Review)
}

\author{
JI UN KANG ${ }^{1}$ and SUN HOE KOO ${ }^{2}$ \\ ${ }^{1}$ Department of Biomedical Laboratory Science, Korea Nazarene University, Cheonan; ${ }^{2}$ Department of \\ Laboratory Medicine, Chungnam National University College of Medicine, Daejeon, Republic of Korea
}

Received February 20, 2012; Accepted April 16, 2012

DOI: $10.3892 /$ ijmm.2012.988

\begin{abstract}
Microarray-based cytogenetics is revealing the tremendous fluidity and complexity of the human genome, and is starting to illustrate the implications of genomic variability with respect to human health and disease. In the last few years, the robustness of array-based technologies has provided accurate diagnosis and appropriate clinical management in a timely and efficient manner for identifying genomic defects of congenital and developmental abnormalities including developmental delay (DD), intellectual disability (ID), autism spectrum disorders (ASD) and/or multiple congenital anomalies (MCA). The implementation of this technology in these categories of disorders has been thoroughly evaluated and is now recommended as a first-line diagnostic approach for clinically suspected genetic disorders. However, clinical application of array-CGH in postnatal evaluation raises the debate of whether array-CGH will replace traditional cytogenetics in the near future and whether there is still a role for karyotyping and FISH. In this article, we therefore review the current status of array-based technology use for postnatal diagnosis and predict that it will replace standard cytogenetics as a first-line test for clinical evaluation in these population groups.
\end{abstract}

\section{Contents}

1. Introduction

2. Classic cytogenetics analysis

3. Molecular cytogenetics analysis

4. Fluorescence in situ hybridization (FISH)

5. Comparative genomic hybridization (CGH)

6. Microarray-based comparative genomic hybridization (array CGH)

7. Postnatal diagnostic applications of array $\mathrm{CGH}$

8. Conclusions and future perspectives

Correspondence to: Dr Sun Hoe Koo, Department of Laboratory Medicine, Chungnam National University College of Medicine, 640 Daesadong, Jung-Gu, Daejeon 301-721, Republic of Korea

E-mail:shkoo@cnu.ac.kr

Key words: cytogenetic analysis, microarray technologies, postnatal diagnosis

\section{Introduction}

Cytogenetics refers to studies of the cellular aspects of heredity, especially the description of chromosome structure and the identification of genomic aberrations that cause disease (1). It has provided the clues for solving fundamental biological questions, such as the nature of inherited syndromes, the genomic changes that are involved in tumorigenesis and the three-dimensional organization of the human genome (2-5). Over the years, cytogenetic techniques evolved and became part of routine laboratory testing, providing valuable diagnostic and prognostic information in congenital and developmental abnormalities.

Microarray-based CGH merges molecular diagnostics with traditional chromosome analysis and is transforming the field of clinical cytogenetics. Evaluation of copy number variation by microarray analysis has significant advantages over standard metaphase karyotyping and is quickly becoming the primary means of postnatal genetic evaluation of patients with developmental delay (DD), intellectual disability (ID), autism spectrum disorders (ASD) and/or multiple congenital anomalies (MCA) $(6,7)$. The use of microarray technologies in these categories of disorders is emerging and promises higher sensitivity for several highly penetrant, clinically severe microdeletion and microduplication syndromes. However, clinical application of microarrays for routine postnatal diagnosis is still being investigated.

In this article, we present a brief overview of how the field is moving away from traditional methods towards molecular genetics approaches for the detection of pathogenic genomic imbalances and highlight the potential utility of the microarray technologies for clinical evaluation of patients with DD, ID, ASD and/or MCA.

\section{Classic cytogenetics analysis}

To understand the importance of microarray technologies to clinical practice, it is helpful to look at these advances in the context of the evolution of clinical cytogenetics.

Chromosome banding analysis was developed in the 1970s and is recognized as the gold standard for the diagnosis and prognosis of congenital and acquired disorders. It has been used for scanning the genome for aberrations that involve both gains and losses of portions of the genome, as well as 
rearrangements within and among chromosomes. Extensive experience gained in the past decades has shown that karyotyping analysis have proven the causal association between specific chromosomal abnormalities and clinical syndromes observed in individuals with DD/ID, ASD or MCA.

However, investigation by standard metaphase karyotyping is not always sufficient to identify the origin of extra chromosome material or correctly assign other structural chromosomal rearrangements (1). In addition, the limited chromosomespecific banding resolution makes the characterization and correct interpretation of complex and cryptic chromosome aberrations difficult to ascertain and is therefore often imprecise in nature.

\section{Molecular cytogenetics analysis}

Molecular cytogenetics, a new field of cytogenetics, and the product of a combination of cytogenetics and molecular biology, has increased the resolution and diagnostic utility of cytogenetic analysis. It has been repeatedly proven effective in genetic diagnostics and has been recognized as a valuable addition or even alternative to chromosomal banding analysis (8-11). The resolution and level of excellence of these techniques are established against cytogenetic banding analysis, which remains the golden standard in this instance.

The overwhelming majority of molecular cytogenetic techniques are based on hybridization. In the last three decades, molecular cytogenetic techniques based on hybridization applications locating specific, fluorescence-labeled nucleic acid sequences in interphase cells or metaphase chromosomes, have become fast, sensitive, and important complementing tools in genetic diagnostics (12).

The use of variable molecular cytogenetics techniques enhances the thorough interpretation of numerical and complex chromosome aberrations, bridging the gap between conventional chromosome banding analysis and molecular genetics DNA studies (13).

\section{Fluorescence in situ hybridization (FISH)}

FISH is based on the use of chromosome region-specific, fluorescent-labeled DNA probes. The technique offers numerous possibilities for identifying genomic imbalances with great accuracy and has become an important complementing application in genetic diagnostics. The application of FISH permits the determination of the number and location of specific DNA sequences, both on metaphase chromosomes and in interphase nuclei, thus significantly simplifying the preparation and evaluation of samples (10). The use of diverse, locus-specific FISH probes and multicolor assays enhances the thorough characterization of numerical and complex chromosome aberrations regardless of their complexity (14).

However, FISH requires clinical suspicion that a specific locus in the genome has undergone copy-number change and therefore is not applicable for whole genome screening approaches required in a diagnostic setting. Furthermore, FISH analysis on metaphase chromosomes detects only microdeletions, and duplications involving segments smaller than 3-5 Mb may be routinely missed even by FISH of interphase nuclei (11).

\section{Comparative genomic hybridization (CGH)}

CGH represents a variation on FISH technology with the clear advantage of revealing imbalances across the whole genome. In CGH, test and reference genomic DNAs are differentially labeled with fluorochromes and then co-hybridized onto normal metaphase chromosomes. Following hybridization, the chromosomes are scanned to measure the fluorescence intensities along the length of the normal chromosomes to detect intensity ratio differences that subsequently pinpoint to genomic imbalances (15). Differences between the fluorescence intensities along the length of any given chromosome will reveal gains or losses of genomic segments. In this way, a global overview of chromosomal gains and losses throughout the whole genome is available and many studies have proved that $\mathrm{CGH}$ is a useful and reliable technique in the research and diagnostics of both cancer and human genetic disorders.

Although the conventional $\mathrm{CGH}$ analysis allows the entire genome to be scanned for aberrations, owing to the limited resolution $(5-10 \mathrm{Mb})$ of metaphase chromosomes, aberrations such as mosaicism, balanced chromosomal translocations, inversions, and whole genome ploidy changes cannot be detected using this approach. In particular, apparently balanced translocations in patients with abnormal phenotypes may hide deletions both at the breakpoint and elsewhere in the genome. Preliminary data demonstrated that other rearrangements, such as ring chromosomes, may be more complex than anticipated (15). For the detection of such abnormalities, a high-resolution technique is required.

Overall, the resolution at which copy number changes can be detected using these molecular cytogenetic techniques are only slightly higher as compared to conventional karyotyping. Furthermore, all experiments are labor-intensive and timeconsuming, especially when multiple genomic regions are interrogated. Therefore, it is strongly advised to investigate these rearrangements with higher resolution and excellent throughput.

\section{Microarray-based comparative genomic hybridization (array CGH)}

Microarray technology represents the technical convergence of molecular genetics and cytogenetics and is rapidly revolutionizing modern cytogenetics. The technology combines fluorescence techniques with the microarray platform and allows the comparison of DNA content in two differentially labeled genomes, a test genome, and a reference genome. In this way the microarray platform allows the use of thousands of individual DNA sequences from throughout the genome, and provides precise information in a single experiment about the locations of any identified aberrations, thereby it holds a great potential for the analysis of DNA copy number changes in clinical genetics (4). A schematic overview of the microarray-based CGH technique is presented in Fig. 1.

The value of whole-genome array $\mathrm{CGH}$ highlights the interpretation difficulties associated with copy number variations of unclear significance. These changes include both numerical and unbalanced structural abnormalities, such as deletions, duplications, or amplifications at any locus as long as that region is represented on the array. An example of cryptic microduplication detected by array $\mathrm{CGH}$ that could 

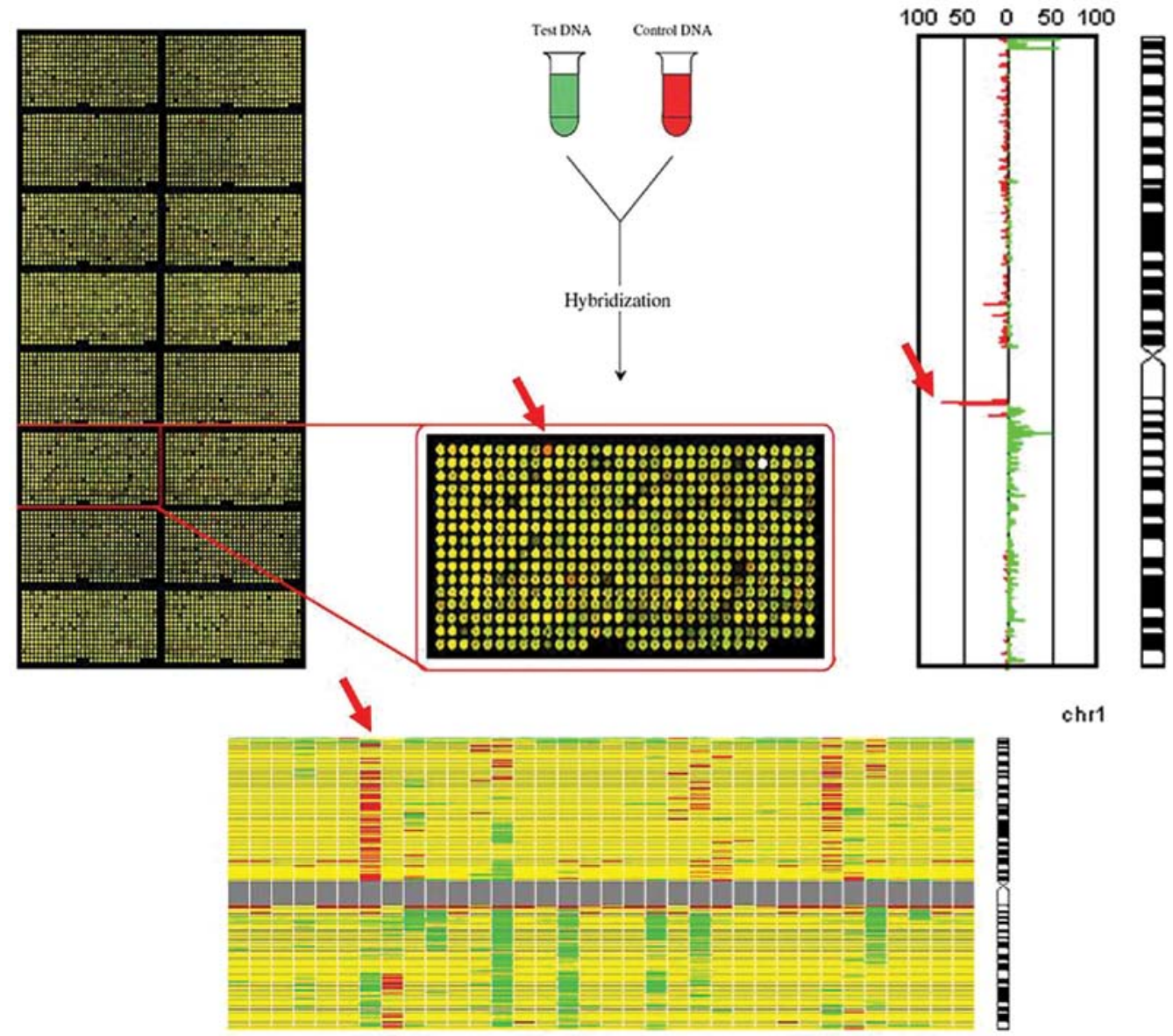

chr1

Figure 1. Schematic overview of microarray CGH technology. Test and control DNA are labeled with a green and red fluorochrome, respectively. Both DNAs are hybridized to cloned DNA fragments that are spotted on a glass slide. Computer imaging assesses the relative fluorescence levels of each DNA for each target on the array. Red spots indicate loss of test DNA (red arrows), green spots indicate gain of test DNA, and yellow spots indicate the presence of equal amounts of test and control DNA. These results can be translated in a high resolution overview of chromosomal copy number changes throughout the whole genome.

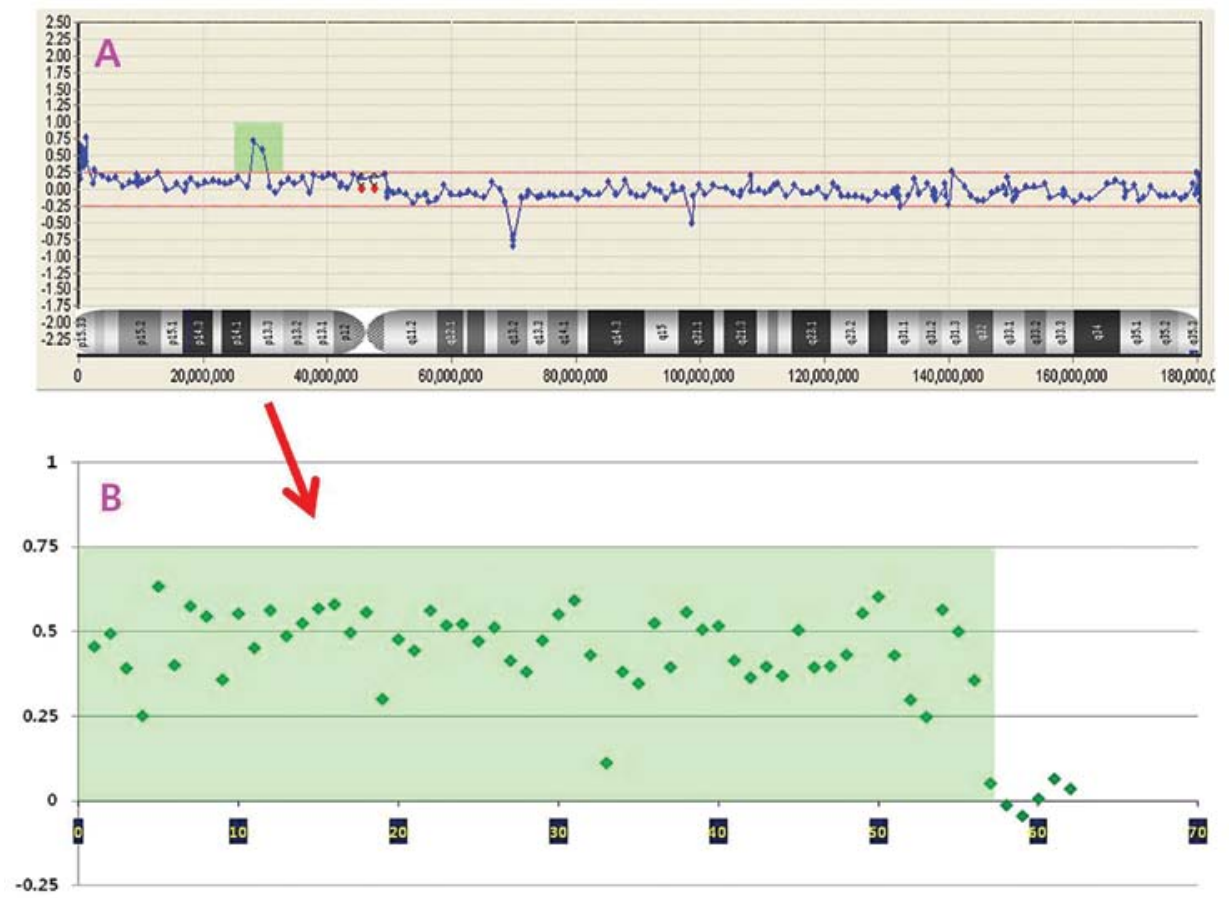

Figure 2. Examples of genomic imbalances detected by array CGH, but not by G-banded karyotyping. (A) Duplication of $4.9 \mathrm{Mb}$ on the short arm of chromosome 5. Increased resolution allowed for the sizing of the segment duplicated from 5p. (B) Arrow points to the close-up view of the duplication at 5p.rom low dose radiation-induced apoptosis. 
Table I. Comparison of technical details between conventional cytogenetic and major molecular approaches.

\begin{tabular}{llc}
\hline Techniques Resolution & Applications & $\begin{array}{c}\text { Specific advantages } \\
\text { of the techniques }\end{array}$
\end{tabular}

$\begin{array}{llll}\begin{array}{l}\text { G-banded } \\ \text { karyotyping }\end{array} & 10-15 \mathrm{Mb} & \begin{array}{l}\text { Identification of numerical } \\ \text { and structural chromosomal } \\ \text { anomalies }\end{array} & \begin{array}{l}\text { Provides global information } \\ \text { in a single assay; simple and } \\ \text { robust procedures }\end{array}\end{array}$

$\begin{array}{ll}\text { FISH } \quad 1-3 \mathrm{Mb} \quad & \begin{array}{l}\text { Identification of the } \\ \text { presence, number of copies } \\ \text { per cell, and localization } \\ \text { probe DNA }\end{array}\end{array}$

$\begin{array}{lll}\begin{array}{l}\text { Multicolor } \\ \text { karyotyping }\end{array} & 1-2 \mathrm{Mb} \quad \begin{array}{l}\text { Detection of subtle rearrangements, } \\ \text { complex translocation, small } \\ \text { marker, ring and double minute } \\ \text { chromosomes }\end{array}\end{array}$

\begin{tabular}{|c|c|c|}
\hline $\mathrm{CGHb}$ & 3-5 Mb & $\begin{array}{l}\text { Interpretation of highly } \\
\text { complex karyotype with } \\
\text { accurate chromosomal } \\
\text { location of imbalance }\end{array}$ \\
\hline
\end{tabular}

Array CGH $\quad 20-150 \mathrm{Mb} \quad$ Identification of cryptic rearrangements (aneuploidy, deletions, duplications, or amplifications) of any locus represented on the array
Fast characterization of marker chromosome origin, high sensitivity

Accurate origin identification of all segments in complex rearrangements; fast characterization of euchromatic marker chromosome content

Whole genome scanning technique without need of proliferating patient material, locus-specific detection of gene amplification, band-specific information on imbalance size

Precise information of whole genome in a single experiment; high-resolution target-specific detection of gene amplification, submicroscopic information on imbalances
Inability to detect minor rearrangements (submicroscopic or cryptic rearrangements)

Structural anomalies cannot be detected in non dividing cells

Inability to detect paracentromeric inversion, duplicatons, microdeletions and cryptic translocation; difficulty to distinguish between artifacts and aneuploidy/polyploidy

Balanced rearrangements and imbalances present in low frequencies remain undetected

Inability to detect balanced translocation, inversion, mosaicism less than $50 \%$, and heterochromatic region

FISH, fluorescence in situ hybridization; CGH, comparative genomic hybridization.

not be detected with the conventional G-banded karyotyping is shown in Fig. 2.

Many studies aimed at assessing the diagnostic capabilities of array $\mathrm{CGH}$ in screening for hidden chromosome aberrations in patients with DD, ID, ASD or MCA who have an apparently normal karyotype. It has been clearly established that for these categories of disorders, the yield of array CGH is significantly higher than karyotyping and any other molecular approaches. The comparison of each technology, its uses and limitations for understanding genomic imbalances is provided in Table I.

\section{Postnatal diagnostic applications of array CGH}

Array $\mathrm{CGH}$ has given the clinician a greater appreciation of variability in the clinical presentation of many well-described conditions and allowed for the discovery of unsuspected imbalances in individuals with congenital and developmental abnormalities. The use of array CGH circumvents the problems associated with conventional cytogenetic testing and it has become a first-tier test in the evaluation of atypical deletions and microduplications in these population groups.

Based on the literature review, the overall rate of detection of genomic abnormalities using a microarray approach in patients with DD, ID or MCA with normal results from prior routine cytogenetic testing is estimated to be between $8-20 \%$ $(4,5,16,17)$. In the study by Dave and Sanger (16) the additional application of array $\mathrm{CGH}$ increased the identification of genomic imbalances by $\sim 8 \%$ in the population of DD with normal conventional banding results. Similar to this result, an array analysis on 13,926 subjects with ID and/or MCA, whom had normal conventional cytogenetic studies, reported an overall diagnostic rate of $10 \%$ for pathogenic genomic imbalances (4). Another retrospective analysis of 36,325 patients with DD also demonstrated that a pathogenic abnormality could be detected in $\sim 19 \%$ of unselected DD/ID patients via genome-wide array approach (5). A review by the International Standard Cytogenomic Array (ISCA) consortium (17) stated that the increased diagnostic yield of array $\mathrm{CGH}$ in individuals 
with congenital anomalies and DD was up to $15-20 \%$, versus the $3 \%$ yield of conventional karyotyping.

During the last few years, improved resolution of array techniques has led to a significant increase in the detection rate of chromosomal aberrations in patients with MR. Many studies aimed to assess the diagnostic capabilities of array CGH to identify hidden chromosome aberrations in patients with MR who had an apparently normal karyotype (18-22). The study of de Vries et al (18) suggested that the diagnostic yield of array $\mathrm{CGH}$ in the general population of patients with MR is at least twice as high as that of standard GTG-banded karyotyping. Also, it has been demonstrated in several other groups of hundreds of patients with idiopathic MR who had an apparently normal karyotype that the diagnostic yield of rearrangements have successfully increased up to $15-20 \%$ after exclusion of inherited anomalies using array platforms (19-21). In addition, the identification of submicroscopic subtelomeric alterations of idiopathic MR patients, as well as the sporadic studies of submicroscopic interstitial chromosomal rearrangements, suggests that a substantial portion of idiopathic MR may be caused by smaller chromosomal rearrangements. These observations make it clear that higher resolution screening techniques for the detection of small deletions or duplications at any chromosomal position will drastically increase the elucidation of human genetic diseases (22).

Many recently published data concluded that array technologies are being increasingly used in patients with DD, ID, ASD or MCA, and microarrays should now be adopted as a first-tier test in place of conventional karyotyping and/or FISH analysis in these population groups $(6,7,23)$. Miller et al (23) recommended a microarray approach as a first-tier test based on studies of 21,698 patients with DD/ID, MCA and/or ASDs in whom the diagnostic yield was $12.2 \%$ higher than that of a G-banded metaphase karyotype. Shen et al (6) also investigated the utility of microarray approach in the evaluation of children with ASD and provided evidence that microarray analysis should be the first-line genetic investigation for children with autism.

From all these considerations, it is clear that a high-resolution array platform covering the whole genome would provide much more informative results than one containing only low coverage limited to postnatal disease-associated regions. Truly balanced rearrangements and low-level mosaicism are generally not detectable by arrays, but these are relatively infrequent causes of abnormal phenotypes in these categories of patients $(<1 \%)$ (23). Bui et al (24) also raised the question whether it matters if the balanced alterations are not detected. For example, carriers of balanced Robertsonian translocations are at risk for uniparental disomy (UPD). Even though, array CGH cannot identify UPD, single nucleotide polymorphism (SNP) arrays can detect regions or chromosomes with copy-neutral absence of heterozygosity, which may indicate an isodisomy (25).

Although, cytogeneticists will continuously require additional training and laboratories must become appropriately equipped, array $\mathrm{CGH}$ holds the promise of being the initial diagnostic tool in the identification of visible and submicroscopic chromosome abnormalities, the latter of which may be missed by routine cytogenetics (11). Further research is required before we can answer the question of an absolute detection rate over conventional karyotyping in these areas on a large cohort that has undergone both karyotyping and analysis using a commercial reproducible array.

\section{Conclusions and future perspectives}

In this review, we present a critical appraisal of the literature to date and provide a summary for clinicians for patients with DD, ID, ASD or MCA. This systematic review provides evidence of the relative advantage of using array testing in postnatal diagnosis, even when the karyotype is normal (26).

Given the potential described in this article, we anticipate array $\mathrm{CGH}$ to be the initial postnatal diagnostic approach for the identification of chromosomal abnormalities and envision that array $\mathrm{CGH}$ will play a major discovery role to reveal the cryptic and/or complex nature of chromosome arrangements in the very near future.

\section{Acknowledgements}

This study was financially supported by the Korea Nazarene University Research Foundation in 2012.

\section{References}

1. Speicher MR and Carter NP. The new cytogenetics: blurring the boundaries with molecular biology. Nat Rev Genet 6: 782-792, 2005.

2. Maciejewski JP and Mufti GJ: Whole genome scanning as a cytogenetic tool in hematologic malignancies. Blood 112: 965-974, 2008.

3. Kang JU, Koo SH, Kwon KC, Park JW and Kim JM: Identification of novel candidate target genes, including EPHB3, MASP1 and SST at 3q26.2-q29 in squamous cell carcinoma of the lung. BMC Cancer 9: 237, 2009.

4. Sagoo GS, Butterworth AS, Sanderson S, Shaw-Smith C, Higgins JP and Burton $\mathrm{H}$ : Array CGH in patients with learning disability (mental retardation) and congenital anomalies: updated systematic review and meta-analysis of 19 studies and 13,926 subjects. Genet Med 11: 139-146, 2009.

5. Hochstenbach R, van Binsbergen E, Engelen J, et al: Array analysis and karyotyping: workflow consequences based on a retrospective study of 36,325 patients with idiopathic developmental delay in the Netherlands. Eur J Med Genet 52: 161-169, 2009.

6. Shen Y, Dies KA, Holm IA, et al: Clinical genetic testing for patients with autism spectrum disorders. Pediatrics 125: 727-735, 2010.

7. Edelmann L and Hirschhorn K: Clinical utility of array CGH for the detection of chromosomal imbalances associated with mental retardation and multiple congenital anomalies. Ann NY Acad Sci 1151: 157-166, 2009

8. Vorsanova SG, Yurov YB and Iourov IY: Human interphase chromosomes: a review of available molecular cytogenetic technologies. Mol Cytogenet 3: 1, 2010.

9. Bejjani BA and Shaffer LG: Application of array-based comparative genomic hybridization to clinical diagnostics. J Mol Diagn 8: 528-533, 2006.

10. Bejjani BA and Shaffer LG: Clinical utility of contemporary molecular cytogenetics. Annu Rev Genomics Hum Genet 9: 71-86, 2008.

11. Shaffer LG and Bejjani BA: Medical applications of array CGH and the transformation of clinical cytogenetics. Cytogenet Genome Res 115: 303-309, 2006.

12. Tönnies H: Modern molecular cytogenetic techniques in genetic diagnostics. Trends Mol Med 8: 246-250, 2002.

13. Lichtenbelt KD, Knoers NV and Schuring-Blom GH: From karyotyping to array-CGH in prenatal diagnosis. Cytogenet Genome Res 135: 241-250, 2011.

14. Bejjani BA, Saleki R, Ballif BC, Rorem EA, Sundin K, Theisen A, Kashork CD and Shaffer LG: Use of targeted array-based CGH for the clinical diagnosis of chromosomal imbalance: is less more? Am J Med Genet A 134: 259-267, 2005. 
15. Vermeesch JR, Fiegler H, de Leeuw N, et al: Guidelines for molecular karyotyping in constitutional genetic diagnosis. Eur J Hum Genet 15: 1105-1114, 2007.

16. Dave BJ and Sanger WG: Role of cytogenetics and molecular cytogenetics in the diagnosis of genetic imbalances. Semin Pediatr Neurol 14: 2-6, 2007.

17. American College of Obstetricians and Gynecologists: ACOG Committee Opinion No. 446: array comparative genomic hybridization in prenatal diagnosis. Obstet Gynecol 114: 1161-1163, 2009.

18. de Vries BB, Pfundt R, Leisink M, et al: Diagnostic genome profiling in mental retardation. Am J Hum Genet 77: 606-616, 2005.

19. Stankiewicz P and Beaudet AL: Use of array CGH in the evaluation of dysmorphology, malformations, developmental delay, and idiopathic mental retardation. Curr Opin Genet Dey 17: 182-192, 2007

20. Vissers LE, de Vries BB, Osoegawa K, et al: Array-based comparative genomic hybridization for the genomewide detection of submicroscopic chromosomal abnormalities. Am J Hum Genet 73: $1261-1270,2003$

21. Shaw-Smith C, Redon R, Rickman L, et al: Microarray based comparative genomic hybridisation (array-CGH) detects submicroscopic chromosomal deletions and duplications in patients with learning disability/mental retardation and dysmorphic features. J Med Genet 41: 24124-24128, 2004.
22. Vermeesch JR, Melotte C, Froyen G, et al: Molecular karyotyping: array CGH quality criteria for constitutional genetic diagnosis. J Histochem Cytochem 53: 413-422, 2005.

23. Miller DT, Adam MP, Aradhya S, et al: Consensus statement: chromosomal microarray is a first-tier clinical diagnostic test for individuals with developmental disabilities or congenital anomalies. Am J Hum Genet 86: 749-764, 2010.

24. Bui TH, Vetro A, Zuffardi O and Shaffer LG: Current controversies in prenatal diagnosis 3: is conventional chromosome analysis necessary in the post-array CGH era? Prenat Diagn 31: 235-243, 2011.

25. Faas BH, van der Burgt I, Kooper AJ, et al: Identification of clinically significant, submicroscopic chromosome alterations and UPD in fetuses with ultrasound anomalies using genome-wide 250k SNP array analysis. J Med Genet 47: 586-594, 2010.

26. Hillman SC, Pretlove S, Coomarasamy A, McMullan DJ, Davison EV, Maher ER and Kilby MD: Additional information from array comparative genomic hybridization technology over conventional karyotyping in prenatal diagnosis: a systematic review and meta-analysis. Ultrasound Obstet Gynecol 37: 6-14, 2011. 